\title{
HOMOGENEOUS AND PRIMARY IDEALS OF R(+)M
}

\author{
Yong Hwan CHO
}

\begin{abstract}
In this short paper, we prove some new properties on homogeneous ideals and primary ideals of $R(+) M$.
\end{abstract}

\section{Introduction}

Let $R$ be a commutative ring with unity and $M$ a unital $R$-module. $M$ is called multiplication module if every submodule $N$ of $M$ has the form $I M$ for some ideal $I$ of $R$. Equivalently, $N=(N: M) M$.

Let $R$ be a commutative ring with unity and $M$ an $R$-module. Consider $R(M)=\{(r, m) \mid r \in R, m \in M\}$ and let $(r, m)$ and $(s, n)$ be two elements of $R(M)$. Define:

1. $(r, m)=(s, n)$ if $r=s$ and $m=n$

2. $(r, m)+(s, n)=(r+s, m+n)$

3. $(r, m)(s, n)=(r s, r n+s m)$

Under these definition, $R(M)$ becomes a commutative ring with unity and $R(M)$ is called the idealization of a ring $R$ and an $R$-module $M$. Sometimes $R(M)$ is also denoted by $R(+) M$. We can find some basic results about $R(M)$ ([6]). For an ideal $I$ of $R, \sqrt{I}=\left\{r \in R \mid r^{n} \in I\right.$ for some positive integer $\left.n\right\}$ and we know that $\sqrt{I(+) M}=\sqrt{I}(+) M$.

A proper submodule $P$ of an $R$-module $M$ is said to be prime if $r m \in P$ for $r \in R, m \in M$ implies that either $m \in P$ or $r \in(P: M)$. The intersection of all prime submodules containing $N$ is denoted by $M-\operatorname{rad} N$.

In section 2 of this paper we prove some basic properties and Theorem 2.6 about homogeneous ideal of $R(M)$ and in section 3 we prove Theorem 3.2

Received July 28, 2009 Accepted September 15, 2009

2000 AMS Subject Classification: 13C13, 13C05,13A15

Key words and phrases : homogeneous ideal, presimplifiable,primary submodule, strongly Laskerian ,multiplication module, idealization 
, Theorem 3.3 about primary ideals of $\mathrm{R}(\mathrm{M})$ and Theorem 3.4 on strongly Laskerian modules

\section{Homogeneous ideals of $\mathbf{R}(+) \mathbf{M}$}

An ideal $H$ of $R(+) M$ is called homogeneous if $H=I(+) N$ where $I$ is an ideal of $R$ and $N$ a submodule of $M$. In this case, $I(+) N=(R(+) M)(I(+) N)=$ $I(+)(I M+N)$ gives $I M \subseteq N$. If $I M \subseteq N$, then $M / N$ is an $R / I$-module and $g: R(+) M \rightarrow R / I(+) M / N$ defined by $g(r, m)=(r+I, m+N)$ is a ring homomorphism and $\operatorname{ker}(g)=I(+) N$ and hence $I(+) N$ is an ideal of $R(+) M$. So we know that $I(+) N$ is an ideal of $R(+) M$ if and only if $I M \subseteq N$. Every ideal of $R(+) M$ of the form $I(+) N$ is homogeneous. However, ideals of $R(+) M$ need not have the form $I(+) N$, that is, need not be homogeneous. $Z(+) 2 Z(2,2)$ is not a homogeneous ideal of $Z(+) 2 Z([2])$.

Proposition 2.1. Let $R$ be a ring and $N$ a submodule of an $R$-module M. Then,

(1) Every ideal of $R(+) M$ containing $0(+) M$ has the form $I(+) M$ for some ideal $I$ of $R$.

(2) Every ideal of $R(+) M$ is contained in $0(+) N$ has the form $0(+) K$ for some submodule $K$ of $N$.

(3) For any homogeneous ideal $H$ of $R(+) M, H=I(+) N$ where $I=\{r \in$ $R \mid(r, m) \in H$ for some $m \in M\}$ and $N=\{m \in M \mid(r, m) \in H$ for some $r \in R\}$.

Proof. (1) Let $H$ be any ideal of $R(+) M$ containing $0(+) M$ and $I=\{r \in$ $R \mid(r, m) \in H$ for some $m \in M\}$. For any $r, s \in I$, there exist $m, n \in M$ such that $(r, m),(s, n) \in H$. Since $(r-s, m-n) \in H, r-s \in I$. Let $a \in R, r \in I$ and $(r, m) \in H$. Since $H$ is an ideal of $R(+) M,(a r, a m)=(a, 0)(r, m) \in H$ and $a r \in I$. Thus $I$ is an ideal of $R$. By the definition of $I$, if $(r, m) \in H$, then $r \in I$ and $(r, m) \in I(+) M$. If $(a, n) \in H$ then $a \in I$ and there exists $m \in M$ with $(a, m) \in H$. Further since $0(+) M \subseteq H,(0, n-m) \in H$ and so $(a, n)=(a, m)+(0, n-m) \in H$. Thus $H=I(+) M$

(2) Let $H$ be any ideal of $R(+) M$ which is contained in $0(+) N$. Put $K=$ $\{k \in N \mid(0, k) \in H\}$. Then $K$ is a submodule of $N$ and $H=0(+) K$.

(3) It is Theorem 25.1 of [6].

Let $N$ be a submodule of an $R$-module $M$ and $I$ an ideal of $R$. The residual 
of $N$ by $I$ is $\left(N:_{M} I\right)=\{m \in M \mid I m \subseteq N\}$. Then it is a submodule of $M$ containing $N([3])$

The following statement is Lemma 1 in [2]. We give the proof for the first assertion.

Proposition 2.2. Let $R$ be a ring and $M$ an $R$ - module. Let $I(+) N$ and $J(+) K$ be homogeneous ideals of $R(+) M$. Then,

$\left(I(+) N:_{R(+) M} J(+) K\right)=(I: J) \cap(N: K)(+)\left(N:_{M} J\right)$

Furthermore, it is a homogeneous ideal of $R(M)$.

Proof. Let $(r, m) \in(I(+) N: J(+) K)$. Then $(r, m) J(+) K \subseteq I(+) N$ and $r J \subseteq I, r K+J m \subseteq N$. Hence $r \in(I: J) \cap(N: K)$. Further $J m \subseteq r K+J m \subseteq$ $N$. So $m \in\left(N:{ }_{M} J\right)$. Thus $(I(+) N: J(+) K) \subseteq(I: J) \cap(N: K)(+)\left(N::_{M}\right.$ $J)$. If $(r, m) \in(I: J) \cap(N: K)(+)(N: M J)$, then $r J \subseteq I, r K \subseteq N$ and $J m \subseteq N$. So, $(r, m) J(+) K \subseteq r J(+) r K+J m \subseteq I(+) N$ and hence $(r, m) \in$ $\left(I(+) N:_{R(+) M} J(+) K\right)$. The proof for the second part is in [2].

Remark 1. From the above Proposition 2.2 we have the following:

(1) If $I(+) N$ is a homogeneous ideal of $R(+) M$, then ann $(I(+) N)=$ $(\operatorname{ann} I \cap \operatorname{ann} N)(+)\left(0:_{M} I\right)$.

(2) If $M$ is a faithful $R$-module and $I(+) N$ is a homogeneous ideal of $R(+) M$, then $\operatorname{ann}(I(+) N)=\operatorname{ann} N(+)\left(0:_{M} I\right)$.

(3) If $M$ is a faithful multiplication module and $I(+) N$ is a homogeneous ideal of $R(+) M$, then $\operatorname{ann}(I(+) N)=\operatorname{ann} N(+)($ annI $) M$.

Proposition 2.3. Let $R$ be a ring and $M$ an $R$-module. For a principal ideal $R(+) M(a, m)$, the following conditions are equivalent;

(1) $R(+) M(a, m)$ is homogeneous.

(2) $R(+) M(a, m)=R a(+)(R m+a M)$.

(3) $(a, 0) \in R(+) M(a, m)$.

(4) there exists $x \in R$ such that $x a=a$ and $x m \in a M$

Proof. (1) $\Rightarrow(2)$. Since $R(+) M(a, m)$ is homogeneous, $R(+) M(a, m)=$ $I(+) N$ for some ideal $I$ of $R$ and a submodule $N$ of $M$ and $I M \subseteq N$. Since $(1,0)(a, m)=(a, m) \in I(+) N, R a(+)(R m+a M) \subseteq I(+) N=R(+) M(a, m)$.

Also $R(+) M(a, m) \subseteq R a(+)(R m+a M)$ is clear.

$(2) \Rightarrow(1)$. Since $R(a M)=a(R M)=a M \subseteq(R m+a M), R(+) M(a, m)$ is homogeneous. 
$(2) \Rightarrow(3)$. Clear.

$(3) \Rightarrow(4)$. Since $(a, 0) \in R(+) M(a, m)$, there exists $(x, n) \in R(+) M$ such that $(a, 0)=(x, n)(a, m)$. Hence $x a=a, x m=-a n \in a M$.

$(4) \Rightarrow(2)$. Suppose that there exists $x \in R, m^{\prime} \in M$ such that $x a=a, x m=$ $a m^{\prime}$, Then $(a, 0)=\left(x a, x m-a m^{\prime}\right)=\left(x,-m^{\prime}\right)(a, m) \in R(+) M(a, m)$. Further $(a, m)-(a, 0)=(0, m) \in R(+) M(a, m)$. Therefore, for any $(s a, t m+a n) \in$ $R a(+)(R m+a M),(s a, t m+a n)=(s, n)(a, 0)+(t, 0)(0, m) \in R(+) M(a, m)$.

A ring $R$ is presimplifiable if for $x, y \in R, x y=x$ implies $x=0$ or $y$ is a unit. Clearly, an integral domain is presimplifiable.

Proposition 2.4. Quaisi local ring is presimplifiable .

Proof. Let $R$ be a quisi local ring. Suppose that $x y=x$ for $x, y \in R$ and $y$ is not a unit in $R$. Then $1-y$ is a unit. If not, $R(1-y) \neq R$ and $R(1-y) \subseteq M, M$ is a unique maximal ideal of $R$. So, $1 \in M$, a contradiction. Therefore, there exists $k \in R$ such that $(1-y) k=k(1-y)=1$. Hence $0=k(x-x y)=k x(1-y)=x(1-y) k=x$.

Proposition 2.5. Let $R$ be presimplifiable. Then $R(+) M(a, m)$ is homogeneous if and only if $a=0$ or $m \in a M$.

Proof. Suppose that $a \neq 0$ and $R(+) M(a, m)$ is homogeneous. By Proposition 2.3, there exists $x \in R$ such that $x a=a, x m \in a M$. Since $R$ is presimplifiable, $x$ is a unit and so $m \in a M$. Conversely, if $a=0$, take $x=0 \in R$ in Proposition 2.3-(4). and if $m \in a M$, take $x=1$ in Proposition 2.3-(4).

Theorem 2.6. Let $R$ be a ring and $M$ an $R$-module. Every ideal of $R(+) M$ is homogeneous if and only if every principal ideal of $R(+) M$ is homogeneous.

Proof. Suppose that every principal ideal is homogeneous and let $H$ be an ideal of $R(+) M$. Then $H=\sum_{\left(a_{\alpha}, m_{\alpha}\right) \in H} R(+) M\left(a_{\alpha}, m_{\alpha}\right)$

$=\sum_{\alpha} R a_{\alpha}(+)\left[R m_{\alpha}+a_{\alpha} M\right]$ (Proposition 2.3)

$=\sum_{\alpha} R a_{\alpha}(+) \sum_{\alpha}\left[R m_{\alpha}+a_{\alpha} M\right]$. So, $H$ is homogeneous. The converse is trivial.

Proposition 2.7. Let $R$ be presimplifiable. Every ideal of $R(+) M$ is homogeneous if and only if $M=a M$ for every $a \neq 0 \in R$. 
Proof. ( $\Rightarrow$ For $a \neq 0 \in R$, and $m \in M, R(+) M(a, m)$ is homogeneous. By Proposition 2.3, there exists $x \in R$ such that $x a=a, x m=a n, n \in M$. Since $R$ is presimplifiable, $x$ is a unit. Hence $m \in a M$ and so $M \subseteq a M$. Thus $M=a M$.

$(\Leftarrow)$ It is enough to show that principal ideal of $R(+) M$ is homogeneous (Proposition 2.6.) If $a=0$, then $R(+) M(a, m)$ is homogeneous by Proposition 2.5 and if $a \neq 0$ then $M=a M$ by assumption. Again by Proposition $2.5, R(+) M(a, m)$ is homogeneous.

REMARK 2. Every ideal of $Z(+) Q$ is homogeneous

\section{Primary ideals of $\mathbf{R}(+) \mathbf{M}$}

A proper submodule $N$ of an $R$-module $M$ is called primary if whenever $r m \in N$, for some $r \in R$ and $m \in M$, then $m \in N$ or $r^{k} \in(N: M)$ for some positive integer $k$.

Proposition 3.1. Let $R$ be a ring and $N$ a submodule of an $R$-module $M$. If $N$ is a primary submodule of $M$, then $(N: M)$ is a primary ideal of $R$ and the converse is true if $M$ is multiplication.

Proof. It comes from Proposition 18 of [8] that if $N$ is a primary submodule then $(N: M)$ is primary.

Let $(N: M)$ be a primary ideal and $M$ a multiplication $R$-module. Clearly, $N \neq M$. Now let $r \in R, n \in M, r n \in N$, and $n \notin N$. Then $r(R n: M) \subseteq$ $(R r n: M) \subseteq(N: M)$. If $(R n: M) \subseteq(N: M)$ then $R n=(R n: M) M \subseteq(N$ : $M) M=N$. So, $n \in N$ and we get a contradiction. Thus $(R n: M) \nsubseteq(N: M)$. Since $(N: M)$ is primary, there exists a positive integer $k$ such that $r^{k} \in(N$ : $M)$.

Theorem 3.2. Let $I$ be a primary ideal of a ring $R$ and $M$ a finitely generated faithful multiplication module over $R$. Then $I M$ is a primary submodule of an $R$-module $M$.

Proof. Let $I M=M$. Then $I M=R M$. Since $M$ is weak-cancellation ([7]Theorem 6.6) and faithful, $I=R$, a contradiction. Therefore $I M \neq M$. Now suppose that $r \in R, m \in M, r m \in I M$ and $m \notin I M . r(R m: M) \subseteq(R r m$ : $M) \subseteq(I M: M)=I+\operatorname{ann}(M)=I$. We know that $(R m: M) \nsubseteq I$. In fact, 
if $(R m: M) \subseteq I$, then $R m=(R m: M) M \subseteq I M$. Hence $m \in I M$ and a contradiction. So, $r(R m: M) \subseteq I,(R m: M) \nsubseteq I$ and since $I$ is primary , $r^{n} \in I=(I M: M)$ for some positive integer $n$.

REMARK 3. Let $M$ be a finitely generated multiplication $R$-module and $I$ an ideal of $R$. Then we always have that $(I M: M)=I+\operatorname{ann}(M)$.

In fact, $I M=(I+\operatorname{ann}(M) M=(I M: M) M$ and since $M$ is weak cancellation, $I+\operatorname{ann}(M)=(I M: M)+\operatorname{ann}(M)$ and so $I+\operatorname{ann}(M)=(I M: M)$.

Theorem 3.3. Let $R$ be a ring and $M$ an $R$-module. Let $I$ be an ideal of $R$ and $N$ a submodule of $M$.

(1) $I(+) I M$ is a primary ideal of $R(M)$, then $I$ is primary and the converse is true if $M$ is finitely generated, faithful,and multiplication.

(2) If $(N: M)(+) N$ is a primary ideal of $R(M)$, then $N$ is a primary submodule of $M$ and the converse is true if $M$ is finitely generated multiplication.

Proof of $(1)$. $(\Rightarrow)$ Since $I(+) I M$ is primary,$I \neq R$. Let $a b \in I, a \notin I$ for $a, b \in R$. Then $(a, 0)(b, 0) \in I(+) I M$ and $(a, 0) \notin(I(+) I M$. Since $I(+) I M$ is primary $(b, 0)^{n} \in I(+) I M$ for some positive integer $n$. Therefore $b^{n} \in I$ and so $I$ is primary.

$(\Leftarrow)$ Since $M$ is finitely generated multiplication, $I+\operatorname{ann}(M)=(I M: M)$ by Remark 3. However since $M$ is faithful, $I=(I M: M)$. Now let $r \in R, n \in$ $M, r n \in I M$ and $n \notin I M$. Then $r(R n: M) \subseteq(R r n: M) \subseteq(I M: M)=I$

However $(R n: M) \nsubseteq I$. Since $M$ is multiplication, $R n=(R n: M) M$ and if $(R n: M) \subseteq I$ then $(R n: M) M \subseteq I M$ and $n \in I M$, contradiction. Since $I$ is primary, there exists a positive integer $n$ such that $r^{n} \in I=(I M: M)$ and so $I M$ is primary. By Proposition 18 of $[1], I(+) I M$ is primary.

Proof of (2). ( $\Rightarrow$ ) Since $(N: M)(+) N$ is primary, $N \neq M$. Let $r m \in N$ and $m \notin N$ for $r \in R$ and $m \in M$. Then $(r, 0)(0, m) \in(N: M)(+) N$ and $(0, m) \notin$ $(N: M)(+) N$. Since $(N: M)(+) N$ is primary, $(r, 0)^{n} \in(N: M)(+) N$ for some positive integer $n$. Hence $r^{n} \in(N: M)$ and $N$ is primary.

$(\Leftarrow)$ Let $I=(N: M)$. Then $N=(N: M) M=I M$ and

$I(+) N=(N: M)(+) N$ is a homogeneous ideal of $R(M)$. Since $M-\operatorname{rad} N=$ $M-\operatorname{rad} I M$ and $N$ is primary, $(I+\operatorname{ann}(M))(+) N=[(N: M)+(0: M)](+) N=$ $(N: M)(+) N=I(+) N$ is primary by Proposition 18 of [1]. 
A primary submodule $Q$ of an $R$-module $M$ is said to be strongly primary if $(\sqrt{(Q: M)})^{n} M \subseteq Q$ for some positive integer $n$. An $R$-module $M$ is called strongly Laskerian if every submodule of $M$ is a finite intersection of primary submodules of $M$ and every primary submodule is strongly primary. A ring $R$ is strongly Laskerian ring if every ideal of $R$ is a finite intersection of primary ideals of $R$ and each primary ideal of $R$ contains a power of its radical ([4],[5]).

Theorem 3.4. Let $R$ be a ring and $M$ an $R$-module. If $R(M)$ is strongly Laskerian, then $R$ is also strongly Laskerian . Assuming further that $M$ is finitely generated, faithful and multiplication then $M$ is strongly Laskerian.

Proof. Suppose that $R(M)$ is strongly Laskerian and $\mathfrak{q}$ a primary ideal of $R$. Then $\mathfrak{q}(+) M$ is a primary ideal of $R(M)$. There exists a positive integer $k$ such that $(\sqrt{\mathfrak{q}(+) M})^{k} \subseteq \mathfrak{q}(+) M$ and further $\sqrt{\mathfrak{q}(+) M}=\sqrt{\mathfrak{q}}(+) M$. Therefore $(\sqrt{\mathfrak{q}(+) M})^{k}=(\sqrt{\mathfrak{q}})^{k}(+)\left((\sqrt{\mathfrak{q}})^{k-1} M\right) \subseteq \mathfrak{q}(+) M$. So, $(\sqrt{\mathfrak{q}})^{k} \subseteq \mathfrak{q}$. Since every ideal is a finite intersection of primary ideals of $R$ ([1]-Corollary 21), $R$ is a strongly Laskerian.

Assume that $M$ is a finitely generated ,faithful and multiplication. Then by Corollary 21 of [1] we know that every submodule of $M$ is a finite intersection of primary submodules of $M$. Hence it is enough to prove that every primary submodule is strongly primary. Let $Q$ be a primary submodule of $M$. Then $(Q: M)$ is primary and $(Q: M)(+) M$ is also primary in $R(M)$. Since $R(M)$ is strongly Laskerian, $(\sqrt{(Q: M)(+) M})^{s} \subseteq(Q$ : $M)(+) M$ for some positive integers. On the other hand, $(\sqrt{(Q: M)(+) M})^{s}$ $=(\sqrt{(Q: M)})^{s}(+)(\sqrt{(Q: M)})^{s-1} M \subseteq(Q: M)(+) M$. Thus $(\sqrt{(Q: M)})^{s} \subseteq$ $(Q: M)$ and since $M$ is multiplication,

$(\sqrt{(Q: M)})^{s} M \subseteq(Q: M) M=Q$ and hence $M$ is strongly Laskerian.

\section{REFERENCES}

1. M.M.Ali, Idempotent and nilpotent submodules of multiplication modules, Comm.in Algebra vol.36 (2008), 4620-4642.

2. __ Idealization and theorem of D.D.anderson, Comm.Algebra vol.35 (2006), 44794501.

3. __ Residual submodules of multiplication modules., Algebra and Geometry vol.46(2) (2005), 405-422.

4. N.Bourbaki, Commutative Algebra, Addison Wesley (1972). 
5. R.Gilmer, Multiplicative ideal theory, Marcel Dekker (1972).

6. J.A.Huckaba, Commutative rings with zero divisors, New York;Marcel Dekker. (1988).

7. A.G.Naum and A.S.Mijbass, Weak cancellation modules, Kyungpook Math.J. vol.37 (1997), 73-82.

8. D.G. Northcott, Lessons on Rings ,modules and Multiplicities, Cambridge University Press (1968).

Department of Mathematics Education

and Institute of Pure and Applied Mathematics,

Chonbuk National University,

561-756,Chonju,Korea 